\title{
THE IMPACT OF MICROELECTRONICS ON PARTICLE DETECTION
}

\author{
Erik H.M. HEIJNE and Pierre JARRON
}

CERN, Geneva, Switzerland

Progress in microelectronics has an impact on silicon detector manufacturing technology and on detector system design. Noise performance, miniaturization and lower cost of hybrid or integrated front-end electronics enable thinner, segmented silicon detectors to be used for a number of new applications.

\section{Introduction}

Semiconductor particle detectors were conceived as miniature energy spectrometers for energetic ionizing particles. The higher the energy, the thicker the detector must be to contain the full energy deposition of the particle. In photon spectrometry, one strives for highest conversion efficiency by using semiconducting materials of high atomic number ( $\mathrm{Ge}, \mathrm{CdTe}, \mathrm{HgI}_{2}$ ) and again by increasing the detector volume as much as possible. The development of these particle detectors occurred in the wake of the general interest in semiconductor devices, but the materials requirements are so stringent that detector manufacturing has become a venerable art, only loosely connected to the semiconductor industry. The production of ultra-pure silicon and germanium is a challenge and the related research has been profitable also to less demanding users through better understanding of the materials properties.

Besides materials requirements, there have also been continuous needs for improvement of the noise performance of the detector-related electronics. These have prompted efforts to design low noise amplifiers and special low noise FET transistors. Such amplifiers now find widespread application, e.g. in optoelectronics.

While progress continues in these traditional areas of materials development and low-noise electronics, there are since 1980 signs of new developments, especially with regard to silicon detectors, which are inspired by the advance in silicon technology for microelectronics. These developments aim at several new applications, where semiconductor detectors can complement or replace other types of detectors, like ionization chambers, bubble chambers, or photographic film. The advantages of the semiconductor detectors are their smaller size, the well-defined geometry, or their instantaneous response in the form of an electrical signal. In this respect, they resemble other types of silicon sensors, which at the moment receive much attention.

\section{The opportunity for silicon sensors}

Pure monocrystalline silicon is probably the purest material commercially available. It is semiconducting at ambient temperatures due to its bandgap of $1.12 \mathrm{eV}$. It contains $5 \times 10^{22}$ atoms $\mathrm{cm}^{-3}$ with a surface density of $2 \times 10^{15} \mathrm{~cm}^{-2}$. The surface atoms react at high temperature with oxygen or nitrogen, forming oxide or nitride layers. The oxide layer is permeable to oxygen atoms, so that the oxidation of $\mathrm{Si}$ can continue at the oxide-silicon interface. This oxide layer is not permeable to common dopant atoms like boron or phosphorus and this gives the possibility of creating locally a $\mathrm{p}-\mathrm{n}$ diode structure. Complicated geometries of such structures can be produced with the help of photolithography and many different devices have been developed. The photolithographic procedure enables very small dimensions and also repeatability, which opens the way to mass production at low cost.

At this moment, the situation can be characterized as an industrial avalanche process: widespread use of silicon and processing apparatus for commercial production of silicon devices has lead to the common availability of such high technology apparatus. This enables further study of the properties of silicon structures and prompts new applications. Automatization needs more silicon, but also produces more. Although the manufacturing of the pure silicon crystals and the various pieces of processing equipment demand vast resources, the production of devices itself does not require a massive infrastructure. The main factors are ingenuity and skill in clean operation.

The systems for information processing have been revolutionized by the application of electronics and silicon technology. The sensors which gather the information could also be produced in silicon technology and also eventually the actuators, which react on the basis of decisions after information processing. Such sensors could deliver directly an electrical signal to the 
information processing part of the system and ultimately both functions could be integrated on the same piece of silicon. Reproducibility and low cost might be possible with silicon sensors.

Several examples of silicon sensors were described by Barth [1], especially a miniature gas chromatograph which has a $10 \mathrm{~s}$ separation time and an accelerometer with on-board MOS amplifiers. These devices not only use electrical, but also mechanical characteristics of silicon. Selective etching is used to "machine" the structures. Etchants have been developed which have different etch rates in different crystal directions, or which can etch preferentially lowly or highly doped silicon. Recent work on silicon sensors has been reported at the Conference, Solid State Transducers 83 [2].

\section{Microelectronics and segmented detector design}

Microelectronics developments influence the detector design in two distinct ways. First of all, improvements in the processing of silicon now make it feasible to manufacture high-quality particle detectors using similar methods as employed for more usual silicon devices [3]. A more elaborate discussion will be given in sect. 5 . In sections 3 and 4 , we discuss the second way in which microelectronics has an impact on particle detection: the availability of better performance, miniaturized, low cost signal processors can lead to a completely different detection system design. The detector itself can be segmented, with parallel processing of signals from the segments. We have shown [4] that in the case of the detection of photons and minimum ionizing particles, there is no charge loss due to the segmentation of the detector.

Although various segmentation geometries might be of interest (e.g. concentric rings, $n \times n$ squares), so far the parallel strip detector has received most attention. The checker-board detector, with perpendicular strips on the front and the rear side [5] has been a predecessor in this approach, although for the energy signal, only a single processor was connected to all strips together.

Large area detectors have a large capacitance, unless they have a very thick depletion layer. The series noise contribution from the preamplifier increases linearly with this capacitance and may become predominant for a capacitance $\geqq 50 \mathrm{pF}$. It is often attractive to use segmented detectors, where the segments have much lower capacitance and hence lower noise. Although one needs a certain number of parallel channels, the cost per channel can be much lower and the detector can be thinner, unless there is also a stopping power requirement.

Counting rate problems can be effectively eliminated by segmented detectors. With small segments and high speed amplifiers, a particle flux of $10^{9} \mathrm{~cm}^{-2} \mathrm{~s}^{-1}$ can be counted. For a thin detector, the time jitter of the rising edge can be below 1 ns, which enables coincidence measurements.

Finally, the main application of silicon microstrip detectors has been recently in position sensitive detection. A precision of $\sim 5 \mu \mathrm{m}$ has been obtained [6] with a $20 \mu \mathrm{m}$ pitch, with interpolating read-out. In the microstrip detector, several particles can be localized simultaneously and fairly low signals can be accepted, because of the low segment capacitance. The noise can be lower than $10 \mathrm{keV}$ fwhm, depending on the amplifier noise.

\section{Low noise front-end electronics}

Generally it is the input transistor which ultimately determines the noise performance of the various frontend signal processors. Junction Field Effect Transistors (JFET) have mostly been used in low noise preamplifiers. Recently, bipolar microwave transistors with very low base spreading resistance $\left(r_{\mathrm{BB}^{\prime}} \leqq 15 \Omega\right)$ have become available and these are competitive as input elements for fast signal processing [7]. For amplifiers in integrated circuits, it would be more attractive to use MOSFET transistors, because of processing compatibility. A short overview will be given of the noise performance of the various types of transistors.

In the high frequency region, the contribution of thermal noise is preponderant. For FET, the equivalent noise voltage $V_{\text {eq }}$ in the frequency interval $\Delta f$ is written [8]

$V_{\mathrm{eq}}=\sqrt{4 k T^{\frac{2}{3}} \frac{1}{g_{\mathrm{m}}} \Delta f}$.

It is inversely proportional to the transconductance $g_{\mathrm{m}}=\partial I_{\mathrm{d}} / \partial V_{\mathrm{gs}}$ with drain current $I_{\mathrm{d}}$ and gate-source voltage $V_{\mathrm{gs}}$. In the special case of MOSFET, $g_{\mathrm{m}}$ is related to the geometry of the device as follows

$g_{\mathrm{m}} \sim \sqrt{W I_{\mathrm{d}} / t_{\mathrm{ox}} L}$,

with (lateral) gate width $W$, gatelength $L$ (from source to drain) and gate oxide thickness $t_{\mathrm{ox}}$, which can be as thin as $50 \mathrm{~nm}$. Note that $L$ is generally much shorter than $W$.

The equivalent thermal noise for a bipolar transistor can be expressed as

$V_{\mathrm{eq}}=\sqrt{4 k T\left(r_{\mathrm{BB}^{\prime}}+1 / 2 g_{\mathrm{m}}\right) \Delta f}$.

The transconductance $g_{m}$ is proportional to the emitter current $I_{\mathrm{e}}$ and the base spreading resistance adds considerably to the noise.

Shot noise is, for the FET, proportional to the gate current and for the bipolar transistor proportional to the base current. This contribution is quite important in 
Table 1

Comparison of state-of-the-art values of parameters for transistors.

\begin{tabular}{|c|c|c|c|}
\hline & Bipolar & JFET & MOSFET ( $5 \mu \mathrm{m}$ lines $)$ \\
\hline $\begin{array}{l}\text { 1/f noise region } \\
\text { corner frequency } f_{c} \\
\text { Equivalent thermal }\end{array}$ & $\leqq 1 \mathrm{kHz}$ & $\leqq 1 \mathrm{kHz}$ & $\leqq 0.1-1 \mathrm{MHz}$ \\
\hline noise voltage per $\sqrt{\mathrm{Hz}}$ & $1 \mathrm{nV}$ & $<1 \mathrm{nV}$ & $10 \mathrm{nV}$ \\
\hline $\begin{array}{l}\text { Drain current/collector current } \\
\text { (dissipation) }\end{array}$ & $0.2 \mathrm{~mA}$ & $5 \mathrm{~mA}$ & $10 \mathrm{~mA}$ \\
\hline $\begin{array}{l}\text { Base current/gate current } \\
\text { (shot noise) }\end{array}$ & $\mu \mathrm{A}$ & $<\mathrm{nA}$ & $<\mathrm{pA}$ \\
\hline $\begin{array}{l}\text { Pulse processing time } \\
\text { of interest }\end{array}$ & $10-100 \mathrm{~ns}$ & $0.1-10 \mu \mathrm{s}$ & $0.1-10 \mu \mathrm{s}$ \\
\hline
\end{tabular}

the bipolar transistors, as can be seen from the comparison in table 1.

The $1 / f$ noise, which basically seems to be related to mobility fluctuations, is much influenced by impurities and processing parameters. For junction transistors, the $1 / f$ noise is negligible for frequencies above $-1 \mathrm{kHz}$. For MOSFET transistors, the $1 / f$ noise is proportional to $t_{\mathrm{ox}} / \sqrt{W L}$ and can be of importance up to $1 \mathrm{MHz}$.

The state-of-the-art performance shown in table 1 is certainly subject to improvements in the near future. Both discrete components and analog subsystems in mixed analog-digital chips are actively studied. Ogawa et al. (Bell Laboratories) state that Si MOSFETs with $0.5 \mu \mathrm{m}$ linewidth are competitive with GaAs MESFETs for high frequency operation at low noise [9]. They obtain $g_{\mathrm{m}}=60 \mathrm{~mA} / \mathrm{V}$. In principle, the Si device can be integrated, but Large Scale Integration (LSI) with $(0.2-0.5) \mu \mathrm{m}$ linewidth is only just beginning. A second example of improved performance of a MOSFET transistor (BF982) is given by Houthoff and Uittenbogaard (Philips) [10]. This $2 \mu \mathrm{m} \mathrm{N-MOS} \mathrm{device} \mathrm{has}$ $t_{\mathrm{ox}}=53 \mathrm{~nm}$ and a reduced $L=1.7 \mu \mathrm{m}$. Using this device as input transistor, we have measured an equivalent noise charge of 200 electrons rms for a drain current of $2.7 \mathrm{~mA}$ (time constant $0.5 \mu \mathrm{s}$ ). The transconductance $g_{\mathrm{m}}=25 \mathrm{~mA} / \mathrm{V}$.

\section{Detector manufacturing}

As mentioned already in the introduction, the silicon used for detectors must be very pure to have a sufficiently long minority carrier lifetime. The resistivity must be $1-50 \mathrm{k} \Omega \mathrm{cm}$, depending on the required detector depletion layer thickness. Around 1970, it had been concluded that the silicon oxidation and diffusion heat treatments nearly always degrade the silicon, whereas the cold surface barrier technology, with some wizardry, yields much better detectors. This situation has remained unchanged for quite a while.
In the meantime, knowledge about silicon oxidation, lifetime preservation, defect engineering, ion implantation and edge profiling has been improved considerably, sometimes by the work of the same people who earlier were trying to make silicon particle detectors. For example, one has been able to reduce the number of unsaturated charge states at the $\mathrm{Si}-\mathrm{SiO}_{2}$ interface by appropriate annealing procedures (e.g. in a hydrogen atmosphere). The total number of surface atoms at the Si surface is $2 \times 10^{15} \mathrm{~cm}^{-2}$. The lowest published interface charge state densities $D_{\mathrm{i}}$ on (100) Si surfaces have fallen from $\sim 10^{12} \mathrm{~cm}^{-2}$ in 1960 to $5 \times 10^{8} \mathrm{~cm}^{-2}$ in 1980 , mainly due to improved technology.

Elsewhere in these Proceedings, Kemmer gives a (slightly simplified) description of his detector manufacturing procedure, which now leads to detectors which have very long minority carrier lifetimes (up to $30 \mathrm{~ms}$ ), low leakage currents and very low noise at room temperature. Commercial high resistivity polished $\mathrm{Si}$ wafers are used in this process, so that one can profit from the highly perfected industrial capability in this field.

It appears that elsewhere similar attempts to produce good quality silicon detectors by ion implantation now yield positive results. Obviously, this approach considerably facilitates the production of segmented detectors.

\section{Conclusion}

Developments in microelectronics are beginning to affect both the silicon detector itself and the related electronics. The first result is the segmented detector in the form of the silicon microstrip detector, which is now enabling new experiments to be conducted, especially in high energy physics.

As will appear clearly in the rest of these Proceedings, many new ideas about detectors, electronics and the integration of both are contemplated. The availability of adequate technology could make progress extremely fast, once the project is well defined. On the 
other hand, there will still be basic limitations. One, which has been passed in silence, is the radiation damage, to which in particular MOS devices are sensitive. Also the microscopic structure of charge generation has hardly been studied experimentally. The new structures create at the same time the need and the opportunity to perform such studies.

It can be expected that the new developments in silicon detectors will lead to a number of new applications of these devices. High precision position detection, high rate counting of (simultaneous) particles or photons and intensity profiles by current integration (synchrotron radiation) are among such new applications.

\section{References}

[1] P.W. Barth, Silicon sensors meet integrated circuits, IEEE Spectrum 18 (September 1981) 33.

[2] The Proceedings of this Conference are published in: Sensors and Actuators, ed., S. Middelhoek, vol. 4 (1983).

[3] J. Kemmer, Nucl. Instr. and Meth. 169 (1980) 499.

[4] E.H.M. Heijne et al., Nucl. Instr. and Meth. 178 (1980) 331.

[5] W.K. Hofker et al., IEEE Trans. Nucl. Sci. NS-13 (1966) 208.

[6] E. Belau et al., Nucl. Instr. and Meth. 214 (1983) 253.

[7] E. Heijne and P. Jarron, IEEE Trans. Nucl. Sci. NS-29 (1982) 405.

[8] P.R. Gray and R.G. Meyer, IEEE J. Solid State Circuits SC-17 (1982) 969.

[9] K. Ogawa et al., Bell System Tech. J. 62 (1983) 1181.

[10] J. Houthoff and T.H. Uittenbogaard, Electr. Components Appl. 5 (1982) 46. 